\title{
Fault Detection Using Dynamic Parity Space Approach
}

\author{
H. Mohamed Basri, K.Lias, W.A.Wan Zainal Abidin, K.M. Tay, H.Zen
}

\begin{abstract}
This paper review the effectiveness of the parity space approach to identify faults or disturbance in a system. The most commonly used is the observer based procedures, and redundancy relationship method. This involves analytical mathematical analysis of geometry and bilinear algebra. Then, technological advances which require complex computation such as artificial intelligence and genetic algorithm had made tremendous improvement to fault Detection and Isolation (FDI) analysis. Dynamic Parity Space Approach was studied for a discrete state-space model. Important data will be extracted using this approach especially for residual generation which is the backbones of FDI analysis. Subsequently, at each time instant $k$, the generated residuals will form a matrix that will define the fault signature. It is remarkable that this approach is proven in this study to be effective in diagnosis and faults isolation.
\end{abstract}

Index Terms-- Fault detection, Linear System, Parity Space Approach,

\section{INTRODUCTION}

$\mathrm{T}$ HIS paper evaluate the Parity Space Approach in detecting faults in a discrete state space system. Fault detection scheme for model based system has been the center of interest in many control processes. Identification of cause of process abnormalities is taken high importance for process automation since it might results into serious damages and unnecessarily machines shutdowns. Before a proper fault diagnosis method was introduced, the task of fault detection and isolation were carried out manually by the hands of human operators. The structure of the systems which incorporates a number of sensors or any measurable instruments exposed itself to absorb disturbances. There is a positive correlation between the number of sensors and the negative impact of disturbances. This method is devoted to study the consistency of a model with respect to noises and disturbances [13]. This approach compute residual vectors which converges to zero when no faults are detected and it turns to non-zero value if faults are presents or the quantity of disturbances goes beyond a certain threshold. In order to extract the residual vectors, a singular decomposition method is used [3].

Faults may affect the behavior of a system and it can evolve a linear system into a highly non linear system due to the addition of noises and disturbances. These external parameters can be considered as faults in the system. The general idea of fault detection analysis is mainly for sensor validation purposes. Parity Space Analysis will highlight the consistency between the model, the actual outputs and the expected outputs [4]. The first procedure of Fault Detection Analysis (FDA) consists of generating a set of residuals that will serve as benchmark to our system to sense faulty behavior. By definition, a system is said to be faulty when the actual behavior does not act in accordance with theoretical outputs of the model [5-7].

\section{FRAMEWORK OF ANALYSIS}

The linear system is defined as the following discrete state space model. As for a preliminary analysis, this approach was idealized and computed for a fault free case. During the simulation, white Gaussian noises will be introduced to carry out the study of the effectiveness of this method to localize faults in a specific system.

$\left\{\begin{array}{l}x(k+1)=A \cdot x(k)+B . u(k) \\ y(k)=C \cdot x(k)\end{array}\right.$

Where $x(k), u(k)$ and $y(k)$ are the system's state vector, input vector, and output vector respectively.

In the case of discrete state space system, the actual state is not only determined by the current state at instant $\mathrm{k}$ but it also integrate the state of the previous instant. Thus, the diagnosis task can be formulated as a recursive problem applied to a window of $r$ iteration. The recursion of equation (1) from instant $\mathrm{k}$ to $k+r$ yields

$x(k+r)=A^{r} . x(k)+\sum_{j=0}^{r-1} A^{r-j-1} B \cdot u(k+j)$

Hence, the output of the system for a number of $r$ iteration can be equated as the following expression. The value of $r$ in this case is fixed by the rank of the observability matrix denoted as $H(r)$ and also known as the parity space order [6]. Assuming that the value of $Y$ and $U$ are known over a finite time of window of size $r$ and integrating Equation (2), gives the expression of the output aver the range of $k$ and $k+r$ iteration.

$y(k+r)=C . x(k+r)=C .\left[A^{r} . x(k)+\sum_{j=0}^{r-1} A^{r-j-1} B . u(k+j)\right]$

The residuals were generated by pre-multiplying matrix of the output by another matrix that will render the system to be insensitive to the state of the system [7]. State $x(k)$ cannot intervene in the analysis because we consider the state to be completely healthy from any disturbances. The product between matrix $\Omega$ and $H(r)$ will create a null space to isolate the state from the analysis

[2]. $\Omega$ is a projection matrix satisfying:

$$
\left\{\begin{array}{l}
\Omega \cdot H(r)=0 \\
\Omega \cdot \Omega^{T}=I_{n} \\
\Omega^{T} \cdot \Omega=I_{n}-\Omega\left(\Omega^{T} . \Omega\right)^{-1} \Omega^{T}
\end{array}\right.
$$

The vector parity then produces residual as.

$P=\Omega \cdot[Y(k, r)-G(r) \cdot U(k, r)]$

Since if there are no faults then this expression just give a combination of random noises. A faulty measurement is detected when this expression tend to a non zero value $y(k)=C . x(k)+\Delta y(k)$ where $\Delta y(k)=\left[\begin{array}{llllll}0 & 0 & \ldots & \Delta y_{j} & \ldots & 0\end{array}\right]$. 